\title{
Flow Rate of Particles through Apertures Obtained from Self-Similar Density and Velocity Profiles
}

\author{
Alvaro Janda, Iker Zuriguel, and Diego Maza \\ Departamento de Física, Facultad de Ciencias, Universidad de Navarra, E-31080 Pamplona, Spain
}

(Received 19 January 2012; published 13 June 2012)

\begin{abstract}
"Beverloo's law" is considered as the standard expression to estimate the flow rate of particles through apertures. This relation was obtained by simple dimensional analysis and includes empirical parameters whose physical meaning is poorly justified. In this Letter, we study the density and velocity profiles in the flow of particles through an aperture. We find that, for the whole range of apertures studied, both profiles are self-similar. Hence, by means of the functionality obtained for them the mass flow rate is calculated. The comparison of this expression with the Beverloo's one reveals some differences which are crucial to understanding the mechanism that governs the flow of particles through orifices.
\end{abstract}

DOI: 10.1103/PhysRevLett.108.248001

PACS numbers: $45.70 .-\mathrm{n}$

The flow of particles through bottlenecks is a ubiquitous situation in nature. From the canonical example of the sand falling down in a hourglass to a group of cells flowing in microchannels [1], all these processes present remarkable similarities independently of the peculiarities of particles involved in each system. For the case of a silo, the exit rate is mainly determined by the ratio between the outlet size $D$ and the particles diameter $d$. Although a general expression for the mass flow rate is still lacking, a first order approach is

$$
W=C \rho_{b} \sqrt{g}(D-k d)^{n+1 / 2} .
$$

This equation was derived by Beverloo [2] from a dimensional analysis of the magnitudes involved in the process. In Eq. (1), $n=1(n=2)$ for the two-dimensional (3D) case, $C$ is a dimensionless constant related to the material properties, $\rho_{b}$ is the bulk density, and $k d$ is a geometrical factor related with an effective outpouring section smaller than the real size of the outlet [3]. The $\sqrt{g}$ term is justified assuming the existence of a region above the orifice below which the grains fall freely under the gravity. This hypothetical region was introduced by Hagen [4], and lately developed by Brown and Richards [5] under the name of "free fall arch." Originally the shape of this region was proposed as parabolic [4], while other authors considered it as a hemispherical dome [6]. Although Beverloo correlation fits very well the flow rate for big orifices, it fails for small ones where clogging is possible $[7,8]$. This has been experimentally proved by Mancok et al. [9] who proposed a new empirical expression to fit the flow rate:

$$
W=C^{\prime} \sqrt{g} \rho_{b}\left[1-\frac{1}{2} e^{-b(D-d)}\right](D-d)^{n+1 / 2},
$$

where $C^{\prime}$ and $b$ are fitting constants. This new correlation includes two important differences with respect to the Beverloo's expression: (a) the flow of grains vanishes just when the outlet size equals the particle diameter, (b) an exponential factor is included to adjust the flow rate in the whole range of exit sizes. The authors suggest that this factor could be related with the lower density of the material near the exit.

Despite the huge number of works related to this issue, there is a lack of experimental evidence on the mentioned empirical explanations. Therefore, in this Letter we study systematically the flow of a granular medium through an orifice placed at the bottom of a two-dimensional silo. The geometry of the setup allows us to measure the volume fraction and the velocity profile at the outlet by means of high-speed motion analysis. We find that the profiles are self-similar in the whole range of explored apertures, even in the region where clogging is observed. Finally, we calculate an analytic expression for the mass flow rate which reproduces the experimental results.

The experimental device consists on a two-dimensional rectangular silo ( $800 \mathrm{~mm}$ high and $200 \mathrm{~mm}$ width) made by two glass plates with two stainless steel strips conforming the lateral walls. The granular material used are monodisperse stainless steel spheres $d=1.00 \pm 0.01 \mathrm{~mm}$, which warrant that the air effect becomes irrelevant [6]. The gap between the glass sheets is slightly larger than the particles diameter $(1.10 d)$ and, as a consequence, the beads arrange themselves in a monolayer. The grains are discharged through a slot situated at the flat bottom of the silo. The size of this aperture can be changed at will and is characterized by the half of its length $R=D / 2$. Finite size effects of lateral walls can be neglected as the dimensions of the container are much larger than the beads and the outlet. A more detailed description of the setup can be found in [10]. The flow rate measurements are accomplished in two different ways depending on the size of $R$. When clogging is frequent $(R<4)$, we measure the number of beads fallen until a jam occurs by means of an electronic scale. Moreover, the time that the particles have been flowing is measured with a photogate timer. Then, the flow rate is calculated just by dividing the number of fallen grains by the flowing time. After that, the outflow is resumed with a pressurized air jet aimed to the outlet. In the case of large 


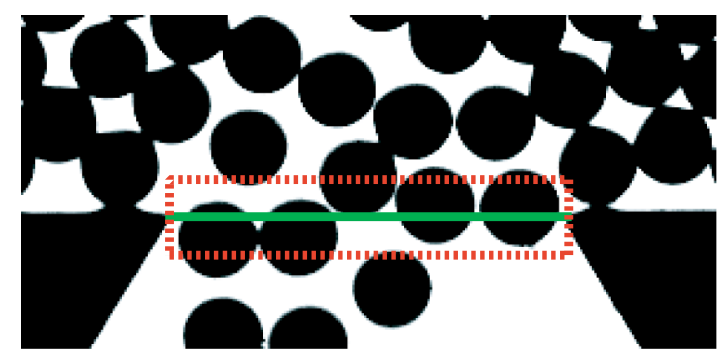

FIG. 1 (color online). Snapshot of the beads flowing through the outlet. Red (green) box (line) shows the position where the volume fraction (velocity) is determined.

$R$, the outpouring is nearly continuous and we measure the flow rate monitoring the mass discharged in constant intervals of time. These measurements are performed several times for each $R$, so finally the mean flow rate $W$ is computed as the arithmetic average of the trials. The velocity and the volume fraction measurements at the exit are performed by high-speed movies of the beads. In the case of the velocity, the tracking of the particles allows us to determine its vertical velocity $v$ just when they pass through the exit (Fig. 1). For the volume fraction, we determine the area of each grain inside a predefined region which is a rectangular box that spans the whole length of the outlet. The height of this region has been fixed to $d$ and is centered at the orifice. It is important to remark that the velocity and volume fraction measurements have been performed after at least five seconds since the first bead crosses the outlet. In this way we can ensure that a steady flow has been reached. The horizontal profiles of both magnitudes are obtained by slicing vertically the outlet in small segments of the same length $(0.3 d)$ where the averages are obtained.

Let us start presenting the velocity profiles for different apertures $R$ ranging from 2.08 to $17.8 \mathrm{~mm}$ [Fig. 2(a)]. Note that the origin of the horizontal axis corresponds to the center of the outlet. Clearly the profiles seem to be the same smooth function independently of the outlet size.
This feature suggests the possibility of rescaling all of them into a single curve. In Fig. 2(b). we show the collapse of all profiles into the function $\frac{v}{v_{c}}=\sqrt{1-(x / R)^{2}}$, where $v_{c}$ is the velocity measured at the center of the orifice. In order to understand the origin of this function let us assume the existence of a parabolic free fall arch of height $h$ spreading on the orifice, $y=h\left[1-\left(\frac{x}{R}\right)^{2}\right]$. Hence, considering that below this dome the particles fall only under the action of the gravity, the vertical velocity just at the orifice can be written as $v^{2}=2 g y$. Therefore, the vertical velocity distribution at the exit is given by the expression $v(x)=$ $\sqrt{2 g h} \sqrt{1-(x / R)^{2}}=v_{c} \sqrt{1-(x / R)^{2}}$. The dependency of $v_{c}$ on $R$ is shown in Fig. 2(c). We fit this data by the expression $v_{c}=\sqrt{2 g \gamma R}$ assuming that the height of the dome must be proportional to the size of the outlet. We find that $\gamma=1.07 \pm 0.015$ which means that the height of the parabolic dome is only $7 \%$ larger than the radius of the orifice. Indeed, writing $v_{c}$ as $\sqrt{2 g R}$ is a very good approximation as it is evident from the dotted line in Fig. 2(c). Accordingly, the velocity profile at the orifice can be written as

$$
v(x)=\sqrt{2 g R} \sqrt{1-(x / R)^{2}} .
$$

As in the case of the velocity, the volume fraction profiles $\phi(x)$ along the exit have been obtained [Fig. 3(a)]. These results are very similiar to the reported by Zulichem, Van Egmond, and De Swart in a three-dimensional case [11]. The first striking observation from these profiles is that, for small orifices, the average value of the density decreases. This seems to be a necessary condition to maintain the material flowing through the outlet. In fact, for radius where clogging events are observed, the flowability condition of the group of particles crossing the outlet would require a strong dilatancy in order to avoid the formation of arches that interrupt the flow. In addition, it is obvious that $\phi(x)$ is not null even near the limit of the orifice and that the volume fraction decreases close to the edges with a well defined functionality. Hence, like the
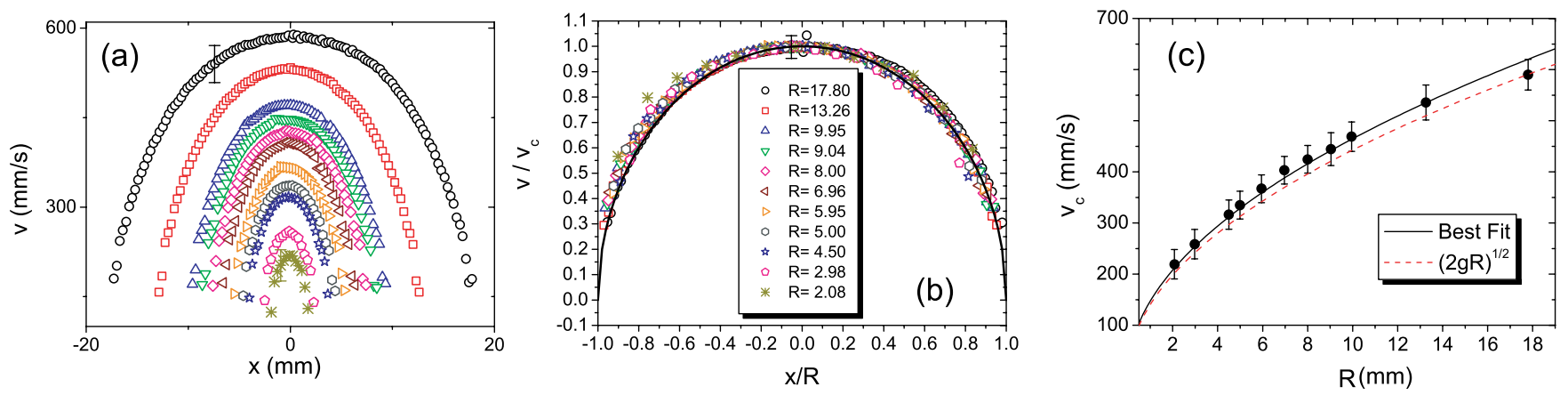

FIG. 2 (color online). (a) Velocity profiles for different outlet sizes. Labels are in the second panel. (b) Normalized velocities and the scaling function introduced in the text (solid line). (c) Dependence of the velocity in the center of the outlet as a function of $R$. The solid line is the best fitting and the dotted $v_{c}=\sqrt{2 g R}$. For clarity, the standard deviation is included only for a single data in panels (a) and (b). Its magnitude is essentially the same for the rest of the data. 

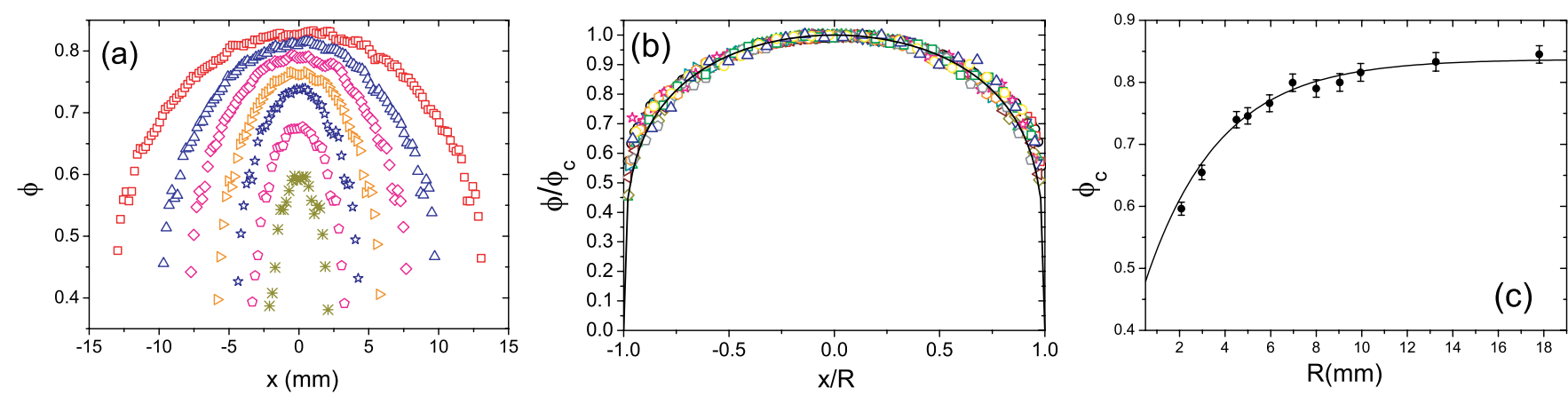

FIG. 3 (color online). (a) Density profiles for different outlet sizes. (b) Collapsed profiles of the data displayed in (a). The symbols are the same that in Fig. 2 and the continuous line is the scaling function introduced in the text. (c) Dependence of the volume fraction in the center of the orifice with $R$. The solid line is the fitting function explained in the text.

velocity, the density profiles can be rescaled by the volume fraction at the center of the outlet $\phi_{c}$ [Fig. 3(b)]. The collapsed data can be fitted conveniently by using a fractional power of the dimensionless radius $\left(1-(x / R)^{2}\right)^{1 / \nu}$, obtaining as best fit $1 / \nu=0.22$. Importantly, the selfsimilarity of the profiles displayed in Fig. 3(a). is a strong indication that the dynamics of the particles flowing through an orifice is not sensitive to the possibility of observe clogging.

Let us now focus on the dependence of $\phi_{c}$ on $R$ which is shown in Fig. 3(c). The behavior seems to correspond to an asymptotic growth which can be fitted by an exponential saturation $\phi_{c}(R)=\phi_{\infty}\left[1-\alpha_{1} e^{-R / \alpha_{2}}\right]$ where $\phi_{\infty}$-the asymptotic value of the volume fraction for big orifices $-\alpha_{1}$ and $\alpha_{2}$ are fitting parameters, whose values are $\phi_{\infty}=0.83 \pm 0.01, \alpha_{1}=0.50 \pm 0.01$, and $\alpha_{2}=3.3 \pm 0.05 \mathrm{~mm}$. In this fitting, the magnitude of $\alpha_{2}$ indicates that the boundaries of the orifice have significative influence on the resulting volume fraction for orifices smaller than 6 times the particle diameter. This length is very similar to the typical scale reported in other dense granular flows [12-14], although the equivalence between both situations is not clear. The meaning of $\alpha_{1}$ is more subtle: when the size of the orifice decreases approaching to the bead diameter, the value of the volume fraction tends to a finite value (0.48) controlled by $\alpha_{1}$. This value is approximately the one obtained assuming an hypothetical situation where the grains fall through the orifice one by one from a height equals to $R(0.52)$. Finally, the magnitude of $\phi_{\infty}$ indicates that the material passing through the orifice has a density lower than the bulk density (larger than 0.90). Remarkably, the value of $\phi_{\infty}$ is very similar to the one obtained for the critical volume fraction at the jamming transition in isotropically compressed granular media [15], $\phi_{\text {jamm }} \cong$ 0.83 . Although the experimental situation is not exactly the same, the agreement between both values is suggestive, indicating that in order to flow continuously through an aperture, the volume fraction should be below the jamming threshold.
Summarizing, the profile for the density just at the exit of the silo, can be written as

$$
\phi(x)=\phi_{\infty}\left[1-\alpha_{1} e^{-R / \alpha_{2}}\right]\left(1-(x / R)^{2}\right)^{1 / \nu} .
$$

Now, assuming that both profiles are stationary, Eqs. (3) and (4) allow us to calculate the flow rate-given in number of particles by time unit-by using arguments of mass conservation:

$$
W=\frac{2}{m} \int_{0}^{R} \sigma \phi(x) v(x) d x,
$$

where $m$ is the mass of the beads and $\sigma$ is the surface density since we are studying the bidimensional projection of the problem. Therefore, the complete expression for the flow rate is

$$
\begin{aligned}
W= & \frac{8}{\pi d^{2}} \sqrt{2 g R} \phi_{\infty}\left[1-\alpha_{1} e^{-R / \alpha_{2}}\right] \\
& \times \int_{0}^{R}\left(1-(x / R)^{2}\right)^{\nu+2 / 2 \nu} d x .
\end{aligned}
$$

By solving this integral [16] the final expression for the mass flow rate is

$$
W=C^{\prime \prime} \sqrt{g} \phi_{\infty}\left[1-\alpha_{1} e^{-R / \alpha_{2}}\right] R^{3 / 2},
$$

where the constant $C^{\prime \prime}=4 \beta\left(\frac{\nu+2}{2 \nu}, \frac{1}{2}\right) / \pi d^{2}$ depends on the diameter of the beads $d$ and on the curvature of the density profile which is fixed by the magnitude of the exponent $1 / \nu$ [17]. In Fig. 4 the experimental flow rate is compared with the prediction of Eq. (5) revealing an excellent agreement. The small discrepancy for small apertures could be related with the existence, at these orifice sizes, of interment arching which give rise to strongly fluctuating flow [18]. Note also that Eqs. (3) and (4) have symmetry of revolution, and hence the scaling with $R^{5 / 2}$ is obtained for the three-dimensional case. Although Eq. (5) has a similar functionality as Eq. (2), there are remarkable differences between both expressions. Indeed, in Eq. (2) the variable $(D-d)$ and the exponential term were introduced $a d$ hoc to fit adequately the experimental observations. On the 


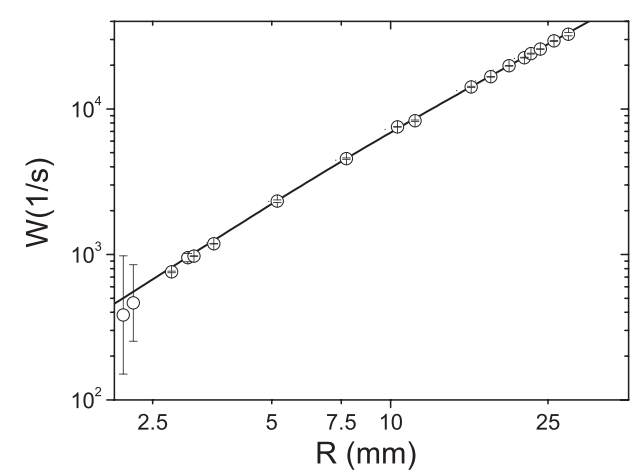

FIG. 4. Flow rate in number of beads per second as a function of $R$. Circles represent experimental results and the continuous line corresponds to Eq. (5).

contrary, all the terms in Eq. (5) have a clear physical meaning provided by the dependence of the velocity and volume fraction near the outlet. Accordingly, Eq. (5) determines the flow rate for any value of the outlet size $R$, without introducing any approximation or extrapolation. Hence, the discharge rate is described by a single expression, even for apertures where clogging is observed and including the hypothetical limit where the system is capable of delivering grains one by one, a possibility recently suggested in [19].

In conclusion, in this work we have derived an expression for the flow rate of particles through orifices determining the velocity and density profiles. Both profiles are self-similar indicating the generality of the mechanism that controls the rate of passing across the aperture. Remarkably, the profiles do not display any significative difference between small orifices (where clogging is commonly observed) and large apertures (where the flow is stationary) stressing such generality. Also, the functionality of both profiles clearly shows that concepts like the empty annulus or the vena contracta introduced by Brown and Richards [5] are not necessary to justify the " $k$ " coefficient in the Beverloo correlation. To our knowledge, these results are the first solid experimental evidence that the mean outpouring velocity scales with the square root of the outlet size, an argument repeatedly used in literature to estimate the mass flow rate of granular materials. This observation is compatible with the existence of an hypothetical singular surface where the bulk stress affecting the particles disappears. Our results also demonstrate that this interface should be parabolic instead of circular as it is commonly considered $[5,6]$. The existence of this hypothetical interface is also in accord with the hypothesissuggested in other works [20-22] - that the bulk dynamics does not have an important influence on the rate of discharge. Although this picture can be hardly assumed by visual inspection of the particle displacements, the collapse obtained for the profiles reveals that the dynamics of the grains could be generalized by introducing an adequate theoretical framework. Finally, and despite of the intrinsic complexity of the flow in the proximities of the outlet region, similarity functions provide a solid evidence that kinetic approach of dense granular matter could be introduced to describe the local rheology of a granular sample flowing through a bottleneck. Such possibility is important not only from a fundamental point of view but also to understand many experimental situations like the recent results about the origin of instabilities in falling granular streams [23]. In fact, velocity and volume fraction profiles lead to gradients inside the falling stream which can play an active role in the reported instabilities.

We thank A. Garcimartín, R. Arévalo, and R. C. Hidalgo for their useful comments and suggestions. This work has been supported by Projects FIS2008-06034-C02-01 and FIS2011-26675 (Spanish Government) and PIUNA (Universidad de Navarra).

[1] J. M. Higgins, D. T. Eddington, S. N. Bhatia, and L. Mahadevan, Proc. Natl. Acad. Sci. U.S.A. 104, 20496 (2007).

[2] W. A. Beverloo, H. A. Leniger, and J.J. Van de Velde, Chem. Eng. Sci. 15, 260 (1961).

[3] R.M. Nedderman, Static and Kinematics of Granular Materials (Cambridge University Press, Cambridge, 1992).

[4] B. P. Tighe and M. Sperl, Granular Matter 9, 141 (2007) (in English).

[5] R.L. Brown and J.C. Richards, Principles of Powder Mechanics (Pergamon Press, Oxford, 1970).

[6] J.E. Hilton and P. W. Cleary, Phys. Rev. E 84, 011307 (2011).

[7] K. To, P. Y. Lai, and H. K. Pak, Phys. Rev. Lett. 86, 71 (2001).

[8] I. Zuriguel, L. A. Pugnaloni, A. Garcimartín, and D. Maza, Phys. Rev. E 68, 030301 (2003).

[9] C. Mankoc, A. Janda, R. Arévalo, J. M. Pastor, I. Zuriguel, A. Garcimartín, and D. Maza, Granular Matter 9, 407 (2007).

[10] A. Janda, A. Zuriguel, I. Garcimartín, A. Pugnaloni, and L. A. D. Maza, Europhys. Lett. 84, 44002 (2008).

[11] D. J. Van Zuilichem, N. D. Van Egmond, and J. G. De Swart, Powder Technol. 10, 161 (1974).

[12] C. T. Veje, D. W. Howell, and R. P. Behringer, Phys. Rev. E 59, 739 (1999).

[13] D. M. Mueth, G. F. Debregeas, G. S. Karczmar, P. J. Eng, S. R. Nagel, and H. M. Jaeger, Nature (London) 406, 385 (2000).

[14] O. Pouliquen and R. Gutfraind, Phys. Rev. E 53, 552 (1996).

[15] T. S. Majmudar, M. Sperl, S. Luding, and R. P. Behringer, Phys. Rev. Lett. 98, 058001 (2007); R. Arévalo, I. Zuriguel, and D. Maza, Phys. Rev. E 81, 041302 (2010).

[16] Performing the change of variable $t=(x / R)^{2}$, the integral can be written as an Eulerian integral of first kind: $\frac{R}{2} \int_{0}^{1}[1-t]^{(\nu+2) / 2 \nu} t^{1 / 2} d t \equiv \frac{R}{2} \beta\left(\frac{\nu+2}{2 \nu}, \frac{1}{2}\right)$ which solution is the Beta function $\beta\left(\frac{\nu+2}{2 \nu}, \frac{1}{2}\right)$.

[17] Certainly, the value of $1 / \nu$ should be somehow related to the intrinsic properties of the grains, friction, restitution 
coefficient, etc., but these details will be discussed elsewhere.

[18] A. Janda, R. Harich, I. Zuriguel, D. Maza, P. Cixous, and A. Garcimartín, Phys. Rev. E 79, 031302 (2009).

[19] A. Janda, D. Maza, A. Garcimartín, E. Kolb, J. Lanuza, and E. Clément, Europhys. Lett. 87, 24002 (2009).

[20] M. A. Aguirre, J. G. Grande, A. Calvo, L. A. Pugnaloni, and J.-C. Géminard, Phys. Rev. Lett. 104, 238002 (2010).
[21] H. Pacheco-Martinez, H. J. van Gerner, and J. C. RuizSuárez, Phys. Rev. E 77, 021303 (2008).

[22] I. Zuriguel, A. Janda, A. Garcimartín, C. Lozano, R. Arévalo, and D. Maza, Phys. Rev. Lett. 107, 278001 (2011).

[23] Y. Amarouchene, J.-F. Boudet, and H. Kellay, Phys. Rev. Lett. 100, 218001 (2008); J. Royer, D. Evans, L. Oyarte, Q. Guo, E. Kapit, and M. Möbius, Nature (London) 459, 1110 (2009). 